\title{
On the Advisability of Removing the Cervix in Performing Hysterectomy for Fibromyomatous Uterine Tumours.*
}

\author{
By John D. MaLcolm, F.R.C.S. (Edin.), \\ Surgeon to the Samaritan Free Hospital, London.
}

A LITrLe over twenty years ago the death rate from the removal of fibroid tumours was decidedly high, but from various causes the mortality has rapidly diminished and now this operation is almost, if not quite, as safe as an ovariotomy. Whether it is wiser to remove the cervix or to leave it is, however, still an open question in this Society and I therefore wish to publish the following notes which seem to me to support the view that the complete operation is the better, the more scientific and the safer.

When the cervix is not removed, the broad ligaments are divided, the ovarian and uterine vessels are ligatured and the uterus is cut away about the level of the inner os, so as to leave anterior and posterior flaps of its tissue, which are brought together and secured in apposition by sutures. The peritoneal edges are then adjusted so as to cover over all the raw surfaces.

Every precaution must, of course, be taken to prevent septic infection, and it is obvious that a special danger of contamination exists at the point of section of the uterus.

Careful attention must also be paid to the arrest of hæmorrhage which is sometimes by no means easy, because, if myomatous growths involve the lower part of the uterus or the cervix, the vessels are often not only enlarged, but numerous and erratic in distribution.

The difficulties of preventing a contamination of the area of operation by the contents of the genital tract do not vary much whether the cervix is left or taken away. But when the cervix is left, even if the raw surfaces are perfectly cleansed, if all the parts are properly adjusted and if hæmorrhage is satisfactorily arrested, the conditions after the operation is finished are, in theory, particularly unfavourable for healing.

The cervix consists of firm tissue and the proper securing of the vessels diminishes the supply of blood to it. So much may this be

* Read before the Obstetrical Society of London, April 3rd, 1907. 
the case that I know of an instance in which the cervix sloughed. Fortunately it separated without doing any harm. In the centre of the cervix, the nourishment of which is thus interfered with, there is a tube of mucous membrane containing many glands, which are not infrequently in a state of chronic inflammation, and no practicable method of cleansing can be relied upon to make such a membrane sterile. Moreover its cut surface is necessarily left in contact with the raw uterine tissue, and the narrowness of the tube interferes with the escape of discharges. It is obvious that this arrangement must be favourable to the development of noxious organisms in the injured parts and thus constitute a source of danger.

Although supra-cervical hysterectomy has undoubtedly proved a very successful procedure, the dangers which I have pointed out must exist so long as that method is adopted, and the following cases, although the patients completely recovered, show that the risks directly due to the method should not be neglected.

In the first case the patient was a nulliparous married woman, aged 40 , who had a soft fibromyoma of the uterus rising nearly to the umbilicus. It had been known to exist for two years and had brought about a condition of extreme anæmia by the profuse hæmorrhages which accompanied its development. The patient had suffered from a feverish illness attributed to tuberculosis of the bases of both lungs in 1894, but she appeared to have recovered completely and to be in all other respects healthy. The tumour and the body of the uterus were removed at the Samaritan Free Hospital on the 23rd of June, 1903, the cervix being left, and there seemed to be no reason when the patient was put back to bed to expect other than a good convalescence. The abdominal incision never showed any sign of irritation and there was no evidence of peritoneal mischief at any time. Nevertheless the patient had the most severe and prolonged attack of phlegmasia dolens that I have seen. From the first the temperature was high. On the fifth day there was pain at the base of the right lung and, on auscultation, friction sounds were detected both before and behind the seat of pain. No râles or evidences of mischief within the lung were detected and the signs of irritation of the pleura ceased after about a week.

On the ninth and tenth days the temperature was above $104^{\circ}$ for seventeen consecutive hours, rising as high as $1062^{\circ} \mathrm{F}$. The patient was then delirious and obviously very ill, but the highest pulse was 126. The temperature fell from $1062^{\circ}$ to $101^{\circ}$ in seven 
hours. There was no immediate explanation of the rapid fall, but about a week later there was a slight escape from the vagina of thick white matter which ceased in a few hours. When an examination was made some fulness and tenderness of the cervix were always present. No swelling was felt at any time beyond or beside the cervix and no appreciable discharge was noted except on the occasion mentioned.

It was not until shortly after the pleural irritation subsided and the temperature moderated that any sign of mischief developed in the legs. First one and then the other calf became swollen and painful and then apparently recovered. With, and following, these manifestations there was a prolonged period of febrile temperature.

Eleven weeks after the operation, the patient again became very ill, the temperature rising nearly to $106^{\circ}$. This was followed by an enormous swelling and much pain in the feet, legs, and thighs, the two sides being about equally affected. There was then some evidence of rectal and bladder irritation, but these symptoms only lasted a few days.

At this time 10 c.c. of antistreptococcic serum were injected subcutaneously without any very obvious effect. A few days later there was a slight general improvement just as there had been before, and this marked the end of the last acute exacerbation. The temperature remained above normal, however, and variable, whilst the pain and swelling subsided only very gradually, and it was not until nineteen weeks after the operation that the patient left the hospital in fairly good condition.

In the summer of 1906 she looked exceedingly healthy and said she could walk six miles without being tired. There was still some tendency to swelling of the legs, which was checked by means of elastic stockings. The patient has recently developed tumours in other parts of the body - a lipoma and a sarcoma apparently involving three costal cartilages--but her further history has no bearing on the subject under discussion. The uterine tumour was not examined microscopically. I had no suspicion that it was other than a simple fibromyoma.

It is, perhaps, important to state that in this case the operation was performed during a week of excessively hot and close weather. I was informed that about the same time, both in the Samaritan Free and in other London hospitals, there had been cases of very high temperature without any obvious cause, and that in one instance death had taken place with an unexplained hyperpyrexia a few days after a hysterectomy. 
If my patient had died from a slightly greater absorption within three or four days of thei operation, I think that very little evidence of mischief would have been found at a post mortem examination, and there might have been no satisfactory explanation of the cause of death.

The pleurisy, the phlegmasia, the tenderness around the cervix, and the absence of any signs of mischief within the peritoneal cavity or in the abdominal incision all pointed, however, to the existence of an insidious form of septic mischief beginning in the uterine stump.

In another case the pathological changes were very similar.

The operation was undertaken on account of a fibro-myoma uteri causing persistent hæmorrhages in a patient whose age was 42 . Only the supra-cervical parts were removed and at first there was no unusual symptom, the bowels being evacuated after two days. The temperature on the third day, instead of falling, rose to $102 \cdot 2^{\circ} \mathrm{F}$., and continued at about the latter level. Nothing, except that the cervix was somewhat swollen, was discovered to account for the prolonged fever until the eighth day when there was a little purulent discharge from the vagina. Its escape was preceded by a further rise of temperature to $103.4^{\circ}$ and immediately afterwards there was a rapid fall of three degrees. The pulse rate kept comparatively at a lower level than the temperature, the highest record being 96 .

The escape of pus was followed almost at once by a slight loss of bright red blood. The hæmorrhage continued for four days and then there was again a discharge of yellow matter, which gradually ceased. Except that the temperature continued to fluctuate, the patient seemed fairly well and the pulse did not rise above 96 . On the tenth day, when the hæmorrhage had begun, 10 c.c. of antistreptococcic serum were injected. This treatment did not seem to have any useful effect. The cervix became less swollen, but a steady escape of blood was taking place.

On the eighteenth day the temperature rose to $105^{\circ} \mathrm{F}$., the highest pulse rate at this time being 100 . The patient had for some days complained of severe and increasing pain over the liver in the midaxillary line and there was considerable œdema of the subcutaneous tissues at the seat of pain. As the patient lay on her back the centre of this swelling was about $5 \mathrm{in}$. below the completely healed puncture wound caused by injecting the serum. The patient was put under an anæsthetic and an examination showed that the cervix was quite mobile, the whole pelvic and abdominal contents seeming to be soft and normal. An incision three inches in length was made 
through the cdematous fat on the right side down to the fascia over the muscles, but $I$ found no bulging or other sign of intraabdominal mischief. I therefore closed the incision and it healed by first intention. The pain ceased, the cedema disappeared and the temperature fell to $99 \cdot 6^{\circ} \mathrm{F}$. two days later. On the third day, the twenty-first after the hysterectomy, the temperature again rose to $104.8^{\circ}$, and this was accompanied by tenderness in many joints, prticularly in the wrists and knees. Sodium salicylate was administered, and after another three days the temperature was normal.

The vaginal discharge had ceased before this time and there was no discovered cause for the amelioration of symptoms apart from the administration of the salicylate, but the improvement was permanent.

In this case it would almost appear that the treatment by injecting antistreptococcic serum might be held responsible for some of the unfavourable conditions which arose. This is important, for at present it is not certain when this method will prove beneficial and in the foregoing cases it was resorted to as being at least very unlikely to do harm. In administering it, every care was taken to prevent septic contamination.

The history of this second case also strongly supports the view that mischief may arise from a form of septic infection spreading from the cervical canal. It seems certain that a small collection of blood was retained and became infected, that the septic products partially escaped, and that then a vessel bled for a few days and the parts healed by granulation.

Undoubtedly there was also a pathological process affecting tissues at a distance from the seat of operation, but there was not sufficient evidence to show whether this was due to an infection from the injured tissues, to an effect of the injection of serum, or to a constitutional disturbance of a rheumatic nature. There was no history of a previous rheumatic attack, although the patient had occasionally complained of pains in her joints.

A third case in which I did not myself perform the operation, but which is instructive in connection with the subject under consideration, has recently come under my observation. The patient was brought to me by her medical attendant thirteen months after hysterectomy had been performed. Her age was 33 . She was of a nervous constitution and the symptoms of the artificially induced menopause - flushings, headaches, etc.-were severe. An additional trouble was a considerable discharge of mucus from the bowel and I gathered that this had been regarded as the chief cause of the 
complaints which the patient had made since the operation. It may have been so, but the cervix had been left in the body and was swollen and tender. On inspection it was red and angry in appearance around the os, from which there was a considerable mucopurulent discharge, and this was said to be increasing in quantity. A sound was easily passed fully half an inch into the cervix, but I did not think it wise to insinuate it further.

It seemed to me that all the symptoms of the "change of life" were exaggerated by the debility and irritation induced by the cervical condition. A catarrh of the rectum with a profuse discharge and sometimes with mucous casts of the intestine is not an uncommon result of an inflammation affecting some part of the genital tract, and it is highly probable that the state of the bowel and the vaginal discharge in the case under consideration were directly due to the presence of the inflamed cervix and that both would have been avoided if a complete hysterectomy had been performed. I thought that possibly a ligature was becoming loose and would be discharged and therefore palliative treatment was recommended for the time, but in such a case the question of removing the cervix by a second operation might have to be considered, and treatment directed to the state of the intestine could not, I think, prove more than palliative until the inflammation in the cervical stump subsided. I understand that the immediate convalescence in this case gave rise to no anxiety.

Although such conditions as those above recorded are, I believe, rare, nevertheless there are cases, and I have already alluded to one, in which death follows a hysterectomy a few days after the operation without any cause being discovered, and such a fatal issue may be due to a sepsis arising by contamination from the contents of the cervix without any local change obvious to the naked eye being induced.

The causes of white leg and of painful œdematous conditions elsewhere as a consequence of an operation are very obscure. I have seen a phlegmasia dolens arising three weeks after a simple abdominal section, from which, in all other respects, the patient appeared to be making a perfectly satisfactory convalescence. It is sometimes held that an extreme degree of anæmia, which existed in the first two cases above recorded, favours the onset of such complications, and it might be argued that the process is not always a septic one, although in many cases it obviously is so. There cannot, however, be any doubt that methods which facilitate the healing of an intra-abdominal 
injury must tend to prevent the subsequent development of this and similar complications.

It may perhaps be held that the complete removal of the uterus only shifts the point of danger from the cervix to the vagina and without doubt the vagina also offers opportunities for septic infection. Since the above was written I have met with a very mild case of phlegmasia dolens after a complete hysterectomy. The patient, whose age was 39 , appeared to be making a satisfactory recovery with the exception that the temperature during the first three weeks, although the highest point recorded was only $1002^{\circ} \mathrm{F}$., did not come down to normal in the evenings. There was no other adverse sign and $I$ thought it well to get the patient up with a view to removing her to the country. Accordingly on the twenty-third day she began to move about. The temperature again rose to $100 \cdot 2^{\circ}$, but the abdominal condition seemed perfect and there was no other indication of danger until the thirty-fifth day when there was a slight, painful swelling of the right leg. Two days later the temperature rose to $103.4^{\circ}$, but within a week it was normal morning and evening and the swelling and pain were gone. After this the patient's progress was good, and I have recently heard that her condition is very satisfactory.

The history of this case favours the view that anæmia is an important predisposing cause of phlegmasia dolens, for before the operation the patient was of extremely unhealthy appearance. Six years earlier she had been told that she had a tumour, but that the state of her health was too bad to permit of operative treatment. In 1906, she came under the care of Mr. Wale of Croydon, who sent her to me. I had no hesitation in advising her to take the risks of an operation as her prospects of improvement without this treatment seemed very remote. That in such a case everything did not progress with complete freedom from complications does not seem to me necessarily to indicate a fault in the method.

The chief disadvantages of the total operation as compared with the partial, are that it generally takes longer to perform and that the manipulation is more difficult. The greater time required may, however, be made up for, to some extent, by the fact that when the cervix is left a very exact arrest of all bleeding points should be obtained; whereas when it is removed if there be some oozing after the patient is put to bed the blood escapes easily and it is therefore harmless. Indeed, unless the loss is dangerous from its quantity, a slight escape may be beneficial by reducing local tension and by washing away infective material. 
The difficulties of manipulation are due to the great length to which the cervix occasionally attains and to the depth in the pelvis at which the surgeon may have to work.

It is sometimes easier to cut away the uterus as low down as possible, and then to seize the rest of the cervix with a volsella and to cut it out. Mr. Bland-Sutton recommends that only the mucous membrane and the parts around it should be removed, a shell of cervical tissue being left. By this plan many of the advantages of the complete operation may be obtained, the chief of these being the removal of the lining membrane of the cervical canal. Mr. BlandSutton's method has not, however, seemed to me easier of execution or more certain of securing a free escape of discharges than that by which there is a complete extirpation of the cervix. Moreover, when this part is much elongated, and $I$ have seen it three inches in length, it must be very difficult to excise the central tube, and I think it must sometimes be almost impossible to be sure that the whole of the mucous membrane has been taken away.

In considering the question as to the best method of performing an operation, the deaths immediately following the procedures under discussion may be important. Our President in a former debate on this matter quoted statistics showing that the mortality from hysterectomy was less when the cervix was taken away than when it was left, the figures being 8.27 per cent. against 8.64 per cent.* My own mortality would compare favourably with either of these figures if I exclude cases operated on by the old-fashioned serre-noeud method. I cannot give exact details because for a considerable period I left the cervix or removed it as $I$ thought fit at the moment, and I find that I have not always been careful to state which method was employed in the cases that recovered. Statistics are, however, in my opinion of no value unless very large numbers are involved, and even then they may be misleading. There are so many circumstances besides the surgical methods that may influence the death rate, and there may also often be a considerable range of legitimate difference of opinion regarding the placing of cases in a table. Moreover bald figures may lead to false conclusions and a consideration of the individual cases leads me to believe that my fatalities are of no value in connection with the particular point raised in this paper. I set aside cases operated on by the serre-noeud method, from which I had eight deaths, six of which have already been 
published in detail.* I also set aside two fatal cases in which malignant tumours were removed and in which it was, of course, right to take the cervix away if it was possible to do so. Besides these, I find only one fatality from the partial operation and five from total hysterectomy. Clearly it might be argued from these figures that the incomplete operation should at least have a further trial and might prove the more successful in my hands. Moreover, in the one fatality from the incomplete operation the body of the uterus was removed only because it was so closely incorporated with a deeply buried ovarian tumour that it was easier to excise than to leave it. The patient died next day from the direct effects of the operation, the duration and severity of which would certainly not have been lessened by an attempt to separate the uterus. As there was no tumour in this case, it might be ignored altogether, leaving my results from the incomplete operation for removal of fibroid tumours perfect so far as the mortality is concerned.

Of the five cases in which death followed a total hysterectomy one might be dismissed on the same grounds, namely, because there was no uterine tumour. The patient was suffering from septicæmia when I removed a large semi-solid ovarian cystoma, which was suppurating in several places and so deeply buried in the broad ligament that it was again easier to remove the uterus than to separate it. This patient also died the day after the operation.

In two cases in which there were large tumours, one a cervical fibroid, the other a tumour of the uterine body as big as a uterus in the seventh or eighth month of gestation, the cervix was 80 expanded that a pan-hysterectomy, or practically that operation, could not be avoided. Both patients died within forty-eight hours of the operation.

Another of these cases is interesting in connection with the fact that a septic infection probably arose from the vaginal wound. The patient was 46 years of age, and I removed the whole uterus and both ovaries at the Samaritan Free Hospital in 1898. She had frequently sufiered from bronchitis with expectoration, but immediately before the operation there was no sign of lung mischief except a few crepitant râles in the left apex. After the operation

\footnotetext{
- Some complicated cases of abdominal section. Case ii., The Lancet, July 18th, 1891 , p. 119.

Illustrations of some modes of death after ovariotomy. Cases iv. and vi., Med. Chir. Trans., 1895.

Twenty-six cases in which an abdominal section has been performed a second time. Cases xii., xxi., and xxii. Med. Soc. Trans., 1896.
} 
the patient was very restless from the first, the pulse was above 120 for two days and the temperature rose to $103.8^{\circ}$ on the second evening.

There was no difficulty in getting the bowels to move or any evidence of peritoneal mischief. On the fifth day the calf of the left leg was swollen and painful and the temperature rose to $1044^{\circ}$, whilst the pulse was 150 . These unfavourable symptoms moderated, but on the sixth day the patient had a troublesome cough, and on the ninth expectoration was profuse. She took food well to the end and showed no signs of peritoneal mischief, but she gradually developed a general septicæmia and died on the sixteenth day, the chest condition being certainly an important cause of the fatal issue; a post mortem examination was not obtained.

Whether the character of the bronchitis was altered by an infection from the contents of the genital tract, similar to that which caused the temporary swelling in the leg, is perhaps an open question, but it does not seem at all improbable.

In the last of these fatal cases death was also due to a lung complication. Much blood was lost in separating adhesions from which the hæmorrhage could not be arrested until the tumour had been released and removed from the pelvis. Several pints of normal saline solution were introduced into a vein with the most gratifying immediate results, but loud râles were heard all over the chest the same evening, and the patient died the next afternoon from acute bronchitis. She had not been subject to any lung trouble before the operation. I am inclined in this case to associate the transfusion with the fatal issue. The method is far too active to be unassociated with dangers of its own. Its employment must, to some extent, depend upon the anæsthetist's opinion of the condition of the patient, and it seems to me at present that there is a too great readiness to resort to this treatment. The tendency is largely fostered in my opinion by erroneous views on the condition of the blood vessels during severe operations.

When I add one case in which a semi-pedunculate uterine tumour was removed from a pregnant uterus, the operation being followed by a miscarriage and death from septicæmia, I have mentioned every case in my. practice of death from operation for the removal of uterine new growths. Of the six cases bearing on the question raised, four died from the excessive severity of the operation, two from lung complications, and I do not think that any weight, either in favour of leaving or of removing the cervix, can be attributed to these results. Another view of the question at issue may be obtained from a 
study of the remote effects of treatment. My colleague, Mr. Alban Doran, has published the results of a series of hysterectomies performed for fibromyomatous tumours, and recorded after an interval of not less than two years. $\mathrm{He}$ is inclined to favour a partial removal, the cervix and even a portion of the mucous membrane of the body of the uterus being left. By this procedure he suggests that a less troubled convalescence is obtained.

The effects of the operation on the progress of the "change of life" are important. But, as was pointed out by Mrs. Florence $N$. Boyd in the debate on Mr. Doran's paper, if we consider how much the troubles of the menopause may vary in women who have not undergone surgical treatment and have had no recognized disease of the genital organs, it seems to me that we ought not to lay too much stress, in regard to this matter, on the results of a series of operations unless the indications are very strongly in favour of one view, and this was not asserted.

Surgical treatment cannot, for example, make a neurotic woman cease to be so, and in those rare cases in which insanity follows an operation on the genital organs I think there is generally a predisposition to mental disorder. I only remember two such cases in my own practice and in each the patient came from an affected stock. In fact, when the condition of a patient after treatment is not satisfactory it may be that the method is not in any way at fault.

I would suggest that women who are otherwise healthy generally make the best recoveries both as regards the immediate and the remote results of a hysterectomy. One of my earliest recollections of abdominal surgery is the remarkable success, as judged by the after results, of many cases in which the old operation, with the use of the serre-noeud and removal of both ovaries and Fallopian tubes, was employed. Similarly, by every modern method or combination of methods as regards taking or leaving the cervix and one or both ovaries, the most gratifying after results are common. When both ovaries have been removed I have seen patients get well so quickly that I am by no means persuaded that the leaving of an ovary or part of an ovary is so important a point as some surgeons believe.

There are patients who are not so fortunate, but one of the worst cases of nervous disturbance after a hysterectomy in my practice was that of a patient aged 54 from whom I removed a very hard fibroid tumour which filled the pelvis and caused much trouble from its weight three years after the periods ceased. The tumour was so calcareous that I could not cut it with a knife. Its removal, the ovaries and cervix being left, was followed by a perfect convales- 
cence, so far as the surgical condition was concerned, but for three years there was almost constant distress, especially in warm weather, from irritation of the skin all over the body. At the end of that time the health of the patient began to improve and she now says that she is very comfortable.

From a consideration of such cases I hesitate to conclude that after-troubles, connected with the artificial induction of the menopause by removal of the uterus, should be attributed altogether to the method of operating, but from a careful study of the whole question I have formed the opinion that the advantages of removing the cervix decidedly outweigh the disadvantages both in theory and in practice.

In theory the removal of the cervix seems to me exceedingly desirable for the reasons which $I$ have stated and in practice the patients, in my experience, recover much more smoothly and comfortably after the total removal of the uterus.

I would, therefore, urge that in performing hysterectomy excision of the cervix should be undertaken whenever there are no very special reasons against it; that, when time permits, the general health of the patient should be made as satisfactory as possible before this operation is performed; and that no woman should be advised to retain a fibroid tumour which has begun to have a definitely prejudicial effect upon her health or which is of any considerable size. These seem to me to be the chief points to be attended to with a view to obtaining a still further success from this which is already one of our most satisfactory operations. 